\title{
Conducting nanocomposites based on polyamide 6,6 and carbon nanofibers prepared by cryogenic grinding
}

\author{
A. Linares*, J.C. Canalda, M.E. Cagiao, T.A. Ezquerra \\ Instituto de Estructura de la Materia (IEM-CSIC), C/Serrano, 12128006 Madrid, Spain
}

\section{A R T I C L E I N F O}

\section{Article history:}

Received 1 March 2011

Received in revised form 3 May 2011

Accepted 15 May 2011

Available online 19 May 2011

\section{Keywords:}

A. Polymer-matrix composites

A. Nanocomposites

A. Carbon fibers

B. Electrical properties

E. Powder processing

\begin{abstract}
A B S T R A C T
Nanocomposites based on polyamide 6,6 and carbon nanofiber have been obtained following a new procedure. It consists of the physical mixing of the polymer matrix, in the form of powder, and the corresponding amount of additive. Then, samples were prepared by compression molding and their structural characteristics, as well as their thermal and electrical properties were determined. The materials present good electrical conductivity at lower percolation thresholds than those corresponding to systems prepared by melt mixing. The study was carried out with two different grain sizes, and the findings are discussed in terms of the different size ratios of polymer to carbon nanofiber.
\end{abstract}

(c) 2011 Elsevier Ltd. All rights reserved.

\section{Introduction}

Conductive composites based on the addition of carbon nanofibers (CNF) to polymeric matrices offer many functional applications in the manufacture of sensors, electromagnetic shielding materials, electrostatically paintable materials, parts for automotive engineering, etc. [1]. Depending on the additive concentration, the electrical conductivity of a composite material varies from that of the polymer matrix to that one of the carbon additive, due to the formation of a percolative network of the conducting additive at a critical concentration [2]. Percolation at low critical concentration is highly desirable in order to remain as close as possible to the mechanical properties of the polymer matrix. The type of polymer, additives themselves, as well as the dispersion method affect the properties of composite materials [21]. Effective utilization CNF in composites with regard to enhancement of electrical conductivity depends primarily on the ability to disperse the nanoadditive homogeneously in the polymer. The first obstacle to be overcome is the destruction of agglomerates due to the intermolecular van der Waals interactions between CNF. Although, as far as industrial applications are concerned the preferred method is melt mixing, it has been reported the breakage of these nanoadditives during processing [3]. Accordingly, a reduction of the aspect ratio has to be taken into account because if the aspect ratio becomes lower, the formation of a percolated conductive network requires higher nanoadditive content. In addition, melt mixing very often leads

\footnotetext{
* Corresponding author. Tel.: +34 915616 800; fax: +34 915855184

E-mail address: alinares@iem.cfmac.csic.es (A. Linares).
}

to polymer degradation, as is the case of polyamide 6,6 (PA66). This polymer presents serious drawbacks (water absorption, low melt viscosity, high melting point, etc.) upon processing in the molten state.

In this paper, we present a study of nanocomposite materials based on PA66 and CNF prepared by an alternative procedure, aiming to obtain nanocomposites with good electrical properties at low loadings, without damaging their mechanical properties.

\section{Experimental}

\subsection{Preparation of PA66 nanocomposites}

Commercial PA66 (DINALON, REPOL, Spain) and commercial grade CNF (GANF, Antolín Ingeniería, Spain) were used as received. The experimental procedure [4] involves:

(1) Obtaining polymer powder by cryogenic milling. Two sieves were used in order to obtain different powder sizes. Particle size distribution was analyzed with a Mastersizer 2000 (Malvern Instruments Ltd.).

(2) Physical mixture of polymer powder with the desired amount of CNF, by a vortex mixer Reax Top (Heidolph).

Different composites were prepared by melt mixing using a rheometer Haake Polylab QC. Temperature was set at $270^{\circ} \mathrm{C}$ and rotor speed at $60 \mathrm{rpm} ; 10 \mathrm{~min}$ mixing time sufficed to generate a steadystate torque response. 
Then, films of about $1 \mathrm{~mm}$ thick were obtained by compression molding at $280{ }^{\circ} \mathrm{C}, 15$ bar pressure, and subsequent cooling down to room temperature.

\subsection{Techniques}

TEM and SEM studies were carried out using a Jeol JEM 2010 and a Hitachi S-300N, respectively. Wide angle X-ray scattering measurements were performed by means of a Seifert XRD 3000 $\theta / \theta$ diffractometer using Ni-filtered $\mathrm{Cu} \quad \mathrm{K} \alpha$ radiation $(\lambda=0.154 \mathrm{~nm})$ at a scanning speed of $0.02 \%$ s. Differential scanning calorimetry study was carried out using a Perkin-Elmer DSC7 system, under nitrogen environment. The temperature was set from 40 to $330^{\circ} \mathrm{C}$, at the heating rate of $20^{\circ} \mathrm{C} / \mathrm{min}$. For the dielectric experiments, a sandwich geometry was used. Measurements were performed, at room temperature, over a frequency window of $10^{-2}-10^{7} \mathrm{~Hz}$, using a Novocontrol spectrometer integrating an ALPHA dielectric interface.

\section{Results and discussion}

Fig. 1 shows TEM micrographs corresponding to the CNF. The characteristic morphology of the nanoadditive consists of a high aspect ratio tubular structure $[5,20]$ made of rolled graphene layers. Fig. 2 shows the particle size distribution corresponding to the two polymer powders. The finest powder exhibits a bimodal distribution with a maximum size around $100 \mu \mathrm{m}$, while the highest one presents a maximum around $450 \mu \mathrm{m}$. Fig. 3A and B shows SEM micrographs of nanocomposites with $1 \mathrm{wt} . \%$ of CNF. In both cases, a tendency to cover the polymer particle in a rather homogenous way is observed.

Fig. 4A shows WAXS patterns corresponding to neat PA66 and some of the nanocomposites, after compression molding. The patterns can be deconvoluted into a broad amorphous halo and three sharper peaks originated by the diffracting planes of the crystalline regions. All samples exhibit the two main peaks corresponding to the $\alpha$ crystalline form of PA66 [6] located at $20.3^{\circ}$ and $23.6^{\circ}$. Additionally, the diffractograms exhibit a peak at $26.2^{\circ}$ corresponding to the reflection from the graphitic layer structure of the CNF. Fig. 4B represents the heating thermograms for the same composites. From these results, it follows that the crystallinity of the polymer matrix does not seem to vary significantly by the presence of the nanoadditives, being the difference among all samples smaller than $0.3 \%$. Moreover, the degree of crystallinity determined by WAXS and DSC are of the same order and similar for all samples.

The electrical conductivity as a function of frequency is shown in Fig. 5. When the nanoadditive content is below $\approx 6 \mathrm{wt} . \%$ for PA66-100 $\mu \mathrm{m}$ and $\approx 4 \mathrm{wt} . \%$ for PA66- $450 \mu \mathrm{m}$, the dependence of

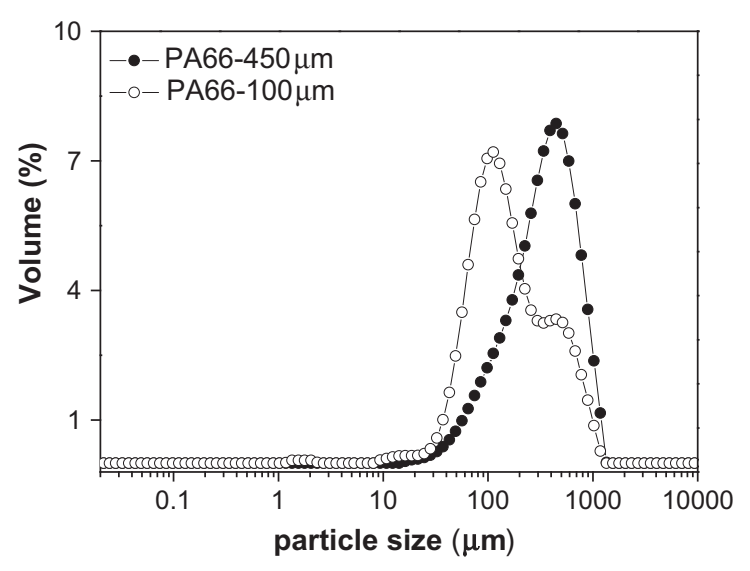

Fig. 2. Particle size distribution of both PA66 powders used in this study.

the electrical conductivity with frequency exhibits the characteristic behavior of an insulating material. This follows from a linear dependence of $\log [\sigma(F)]$ with the logarithm of the frequency with a slope close to 1 . This behavior is similar to that shown by the PA66 matrix. However, for concentrations higher than the abovereferred values, $\sigma(F)$ adopts a different behavior that can be formally described by the so-called universal dynamic response [7] expressed by a law of the type:

$\sigma(F)=\sigma_{\mathrm{dc}}+\sigma_{\mathrm{ac}}=\sigma_{\mathrm{dc}}+A F^{s}$

where $\sigma_{\mathrm{dc}}$ is the frequency independent direct current conductivity and the exponent $s$ fulfills $0<s<1$. This law introduces a critical frequency, $F_{c}$, above which $\sigma(F)=\sigma_{\mathrm{ac}} \propto \mathrm{F}^{s}$. The continuous lines in Fig. 5 correspond to fits of Eq. (1) to experimental data. From these fits, the $\sigma_{\mathrm{dc}}$ values can be extracted. The value of the conductivity at the lowest measured frequency $\left(10^{-2} \mathrm{~Hz}\right)$ has been considered as the $\sigma_{\mathrm{dc}}$ for the insulating samples.

Fig. 6 represents $\sigma_{\mathrm{dc}}$ data as a function of CNF concentration for the two system investigated (conductivity data corresponding to composites prepared from the molten state are also included). In both cases, a characteristic percolative behavior is observed. According to percolation theory, the insulator-conductor transition occurs at the critical concentration at which an infinite cluster of physically connected particles appears. For low concentrations, the conductivity remains at the same level as the insulating matrix. At a certain critical concentration around $6 \mathrm{wt} . \%$ for PA66-100 $\mu \mathrm{m}$ and $4 \mathrm{wt} . \%$ PA66- $450 \mu \mathrm{m}$, the conductivity starts a sudden increase. The dc conductivity above the critical concentration can be analyzed in terms of the percolation theory $[7,8]$ by means:
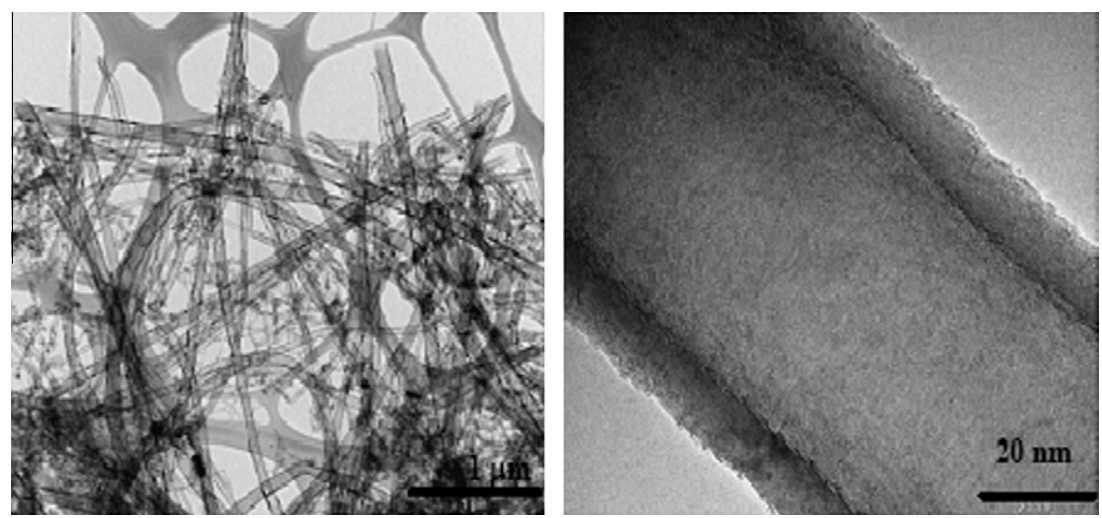

Fig. 1. TEM images of CNF. The background honeycomb-like structure is due to the TEM grid. 

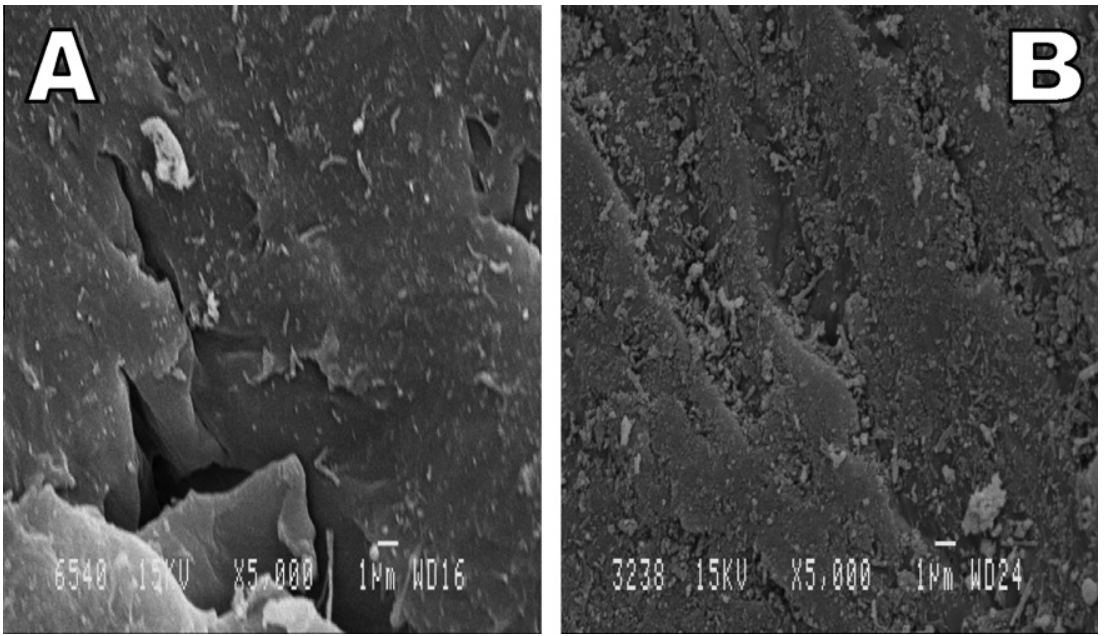

Fig. 3. SEM images of (A) PA66-100 $\mu \mathrm{m}$ and (B) PA66- $450 \mu \mathrm{m}$ with $1 \mathrm{wt} . \%$ of CNF.
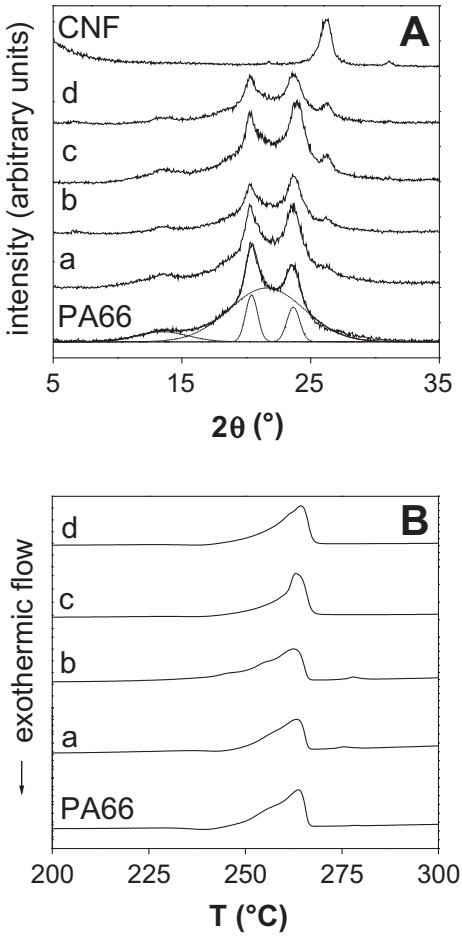

Fig. 4. WAXS patterns (A) and DSC-curves (B) corresponding to: (a) PA66$100 \mu \mathrm{m}+3.5 \% \mathrm{CNF}$; (b) PA66-100 $\mu \mathrm{m}+8 \% \mathrm{CNF}$; (c) PA66-450 $\mu \mathrm{m}+5 \% \mathrm{CNF}$ and (d) PA66-450 $\mu \mathrm{m}+11 \%$ CNF. The different contributions in which WAXS patterns can be decomposed are shown in the figure.

$\sigma_{\mathrm{dc}} \propto\left(\phi-\phi_{c}\right)^{t}$

where $t$ is a critical exponent. Although the critical concentration, $\phi_{c}$, depends on the lattice in which particles are accommodated, $t$ depends primarily on the dimensionality of the percolating system and not on the details of the geometric structures or the interactions [7,8]. Theoretical calculations [9] propose values of $t$ between 1.6 and 2 for three-dimensional systems. The fitting of Eq. (2) to experimental data of Fig. 6 provides $t$ values of around 2.7 and 2.4 with $\Phi_{c} \sim 6$ wt.\% and 4 wt.\% for composites based on PA66$100 \mu \mathrm{m}$ and PA66-450 $\mu \mathrm{m}$, respectively. Although these values are larger than the generally reported ones, they can be considered as acceptable for a percolative model, since composite materials with
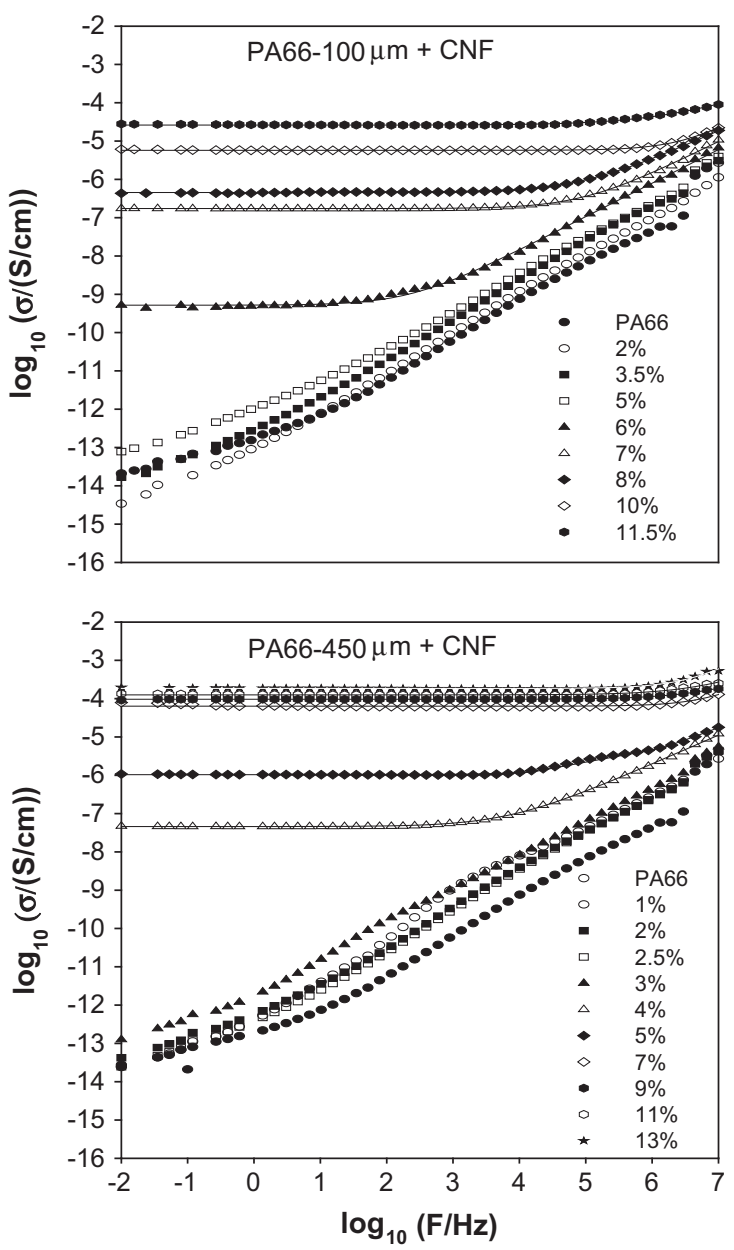

Fig. 5. Broadband electrical conductivity as a function of frequency. The continuous lines represent fits according to Eq. (1).

high values of the critical exponent have been frequently reported in the literature [10], even deduced from numerical simulation methods when an intercluster polarization is assumed [11].

Broadband dielectric measurements also provide information about the ac conductivity as described by the frequency dependent part of Eq. (1). The results of Fig. 5 shows that the electrical 


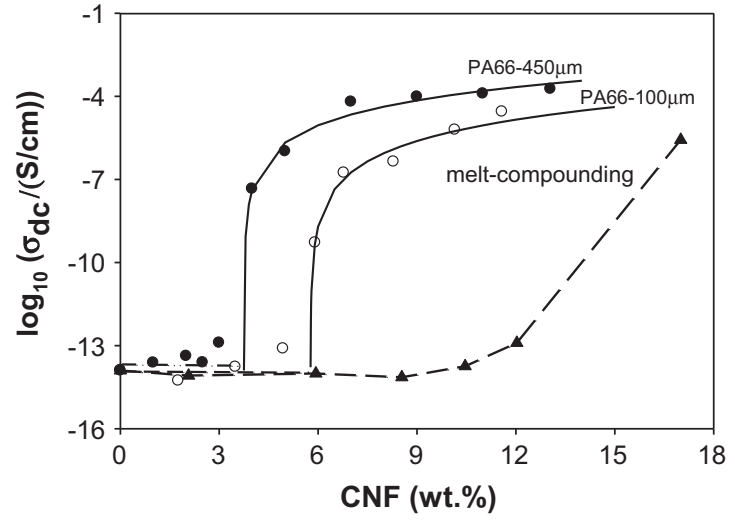

Fig. 6. Electrical conductivity versus nanoadditive concentration. Continuous lines are the fitting of Eq. (2). Data corresponding to composites obtained by melt mixing are also included $(\boldsymbol{\Lambda})$.

conductivity remains constant until a certain critical frequency is reached. Moreover, the higher the dc conductivity, the higher the critical frequency. This effect, which has been observed for several disordered materials [12], is described by:

$F_{c} \propto \sigma_{d c}^{b}$

where the exponent $b$ takes values close to 1 . Fig. 7 shows $F_{c}$ as a function of $\sigma_{\mathrm{dc}}$. The $F_{c}$ data have been calculated as that frequency at which a $5 \%$ increase in conductivity with respect to the corresponding $\sigma_{\mathrm{dc}}$ value is observed. The power law is rather well fulfilled with an exponent $b \approx 0.9$ for both types of composites. At frequencies $F>F_{c}$, the conductivity exhibits a frequency dependence that can be described as $\sigma_{\mathrm{ac}} \propto \mathrm{F}^{s}$. Fig. 8 shows the variation of $F_{c}$ and $s$, as obtained from the fitting of Eq. (1), with CNF content. As seen, $\log F_{c}$ increases linearly with carbon nanoadditive content, whereas $s$-exponent starts from 1 and then a decrease is observed.

By simulation studies Bergman and Imry [13] showed that the variation with frequency, in the percolation concentration vicinity, of the ac conductivity and dielectric constant of a heterogeneous mixture might be described by a power law at frequencies $F>F_{c}$ :

$\sigma_{a c}\left(F, \Phi_{c}\right) \sim F^{x}$

$\varepsilon_{a c}^{\prime}\left(F, \Phi_{c}\right) \sim F^{-y}$

these critical exponents, $x$ and $y$, are supposed to satisfy:

$x+y=1$

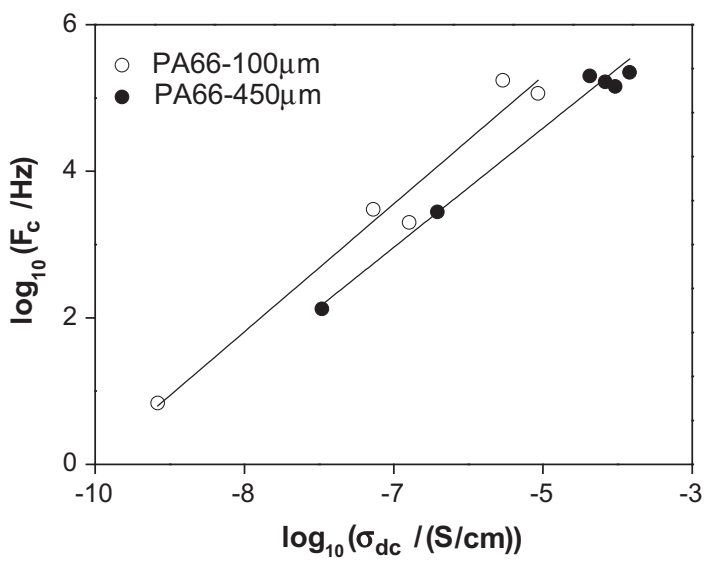

Fig. 7. Logarithm of the critical frequency versus the logarithm of $\sigma_{\mathrm{dc}}$.
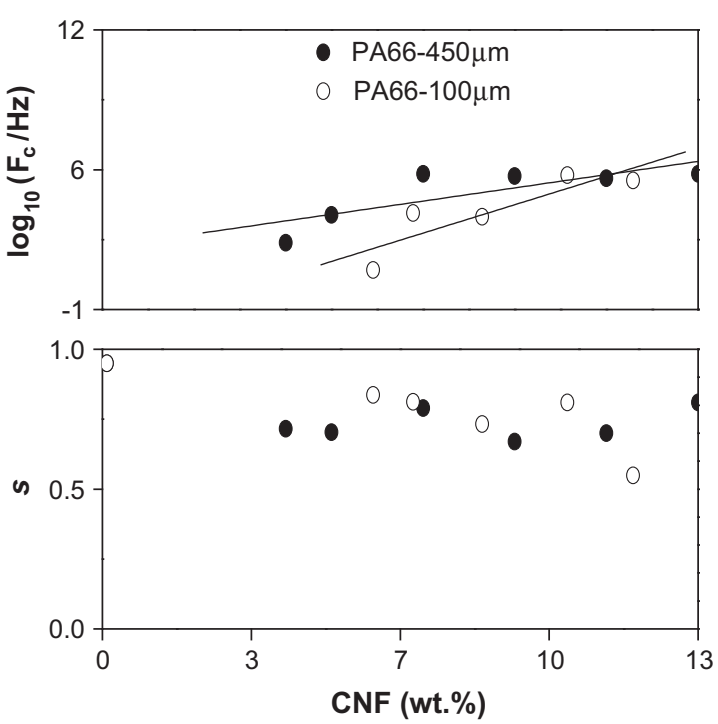

Fig. 8. Logarithm of the critical frequency and exponent $s$ according to Eq. (1) as a function of CNF content.

It is to be noted that the exponent $x$ is equal to $s$ in the Jonscher equation (see Eq. (1)). Fig. 9 shows data of ac conductivity and dielectric constant, versus frequency, near the percolation threshold. The results are in good agreement with the above general scaling relation (Eq. (6)). For three-dimensional materials, it has been proposed $x$ values ranging from $\approx 0.72$, when the frequency dependence of these functions has been attributed to the polarization effects between clusters in the matrix [14], to $\approx 0.58$ when anomalous diffusion within each percolating cluster is proposed [15]. From our results, we conclude that the values found for both systems are closer to the predictions of the intercluster polarization model.

Regarding these results, one aspect to be emphasized is the low percolation thresholds if compared with those reported for similar systems, but prepared by melt mixing $[16,17]$ (see also Fig. 6). Assuming that by this procedure the carbon nanofiber breakage does not take place, or at least it would be not so severe as in the case of melt mixing, a plausible explanation to that fact could be that the PA66 solid particles create volume excluded, reducing

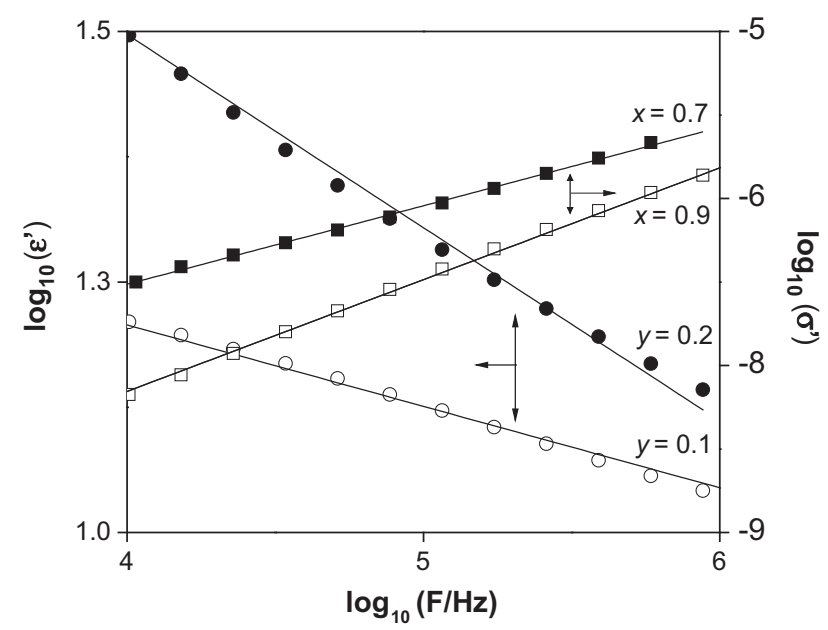

Fig. 9. Dependence of ac conductivity and dielectric constant with frequency near percolation threshold. Open symbols correspond to PA66-100 $\mu \mathrm{m}$ and solid symbols to PA66-450 $\mu \mathrm{m}$ nanocomposites. 
A
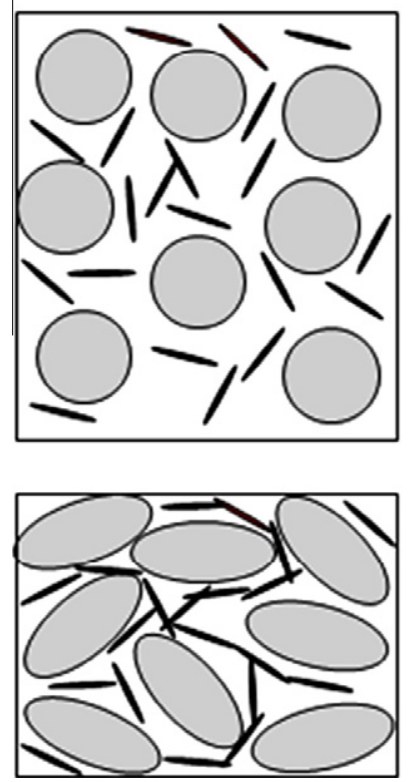

B
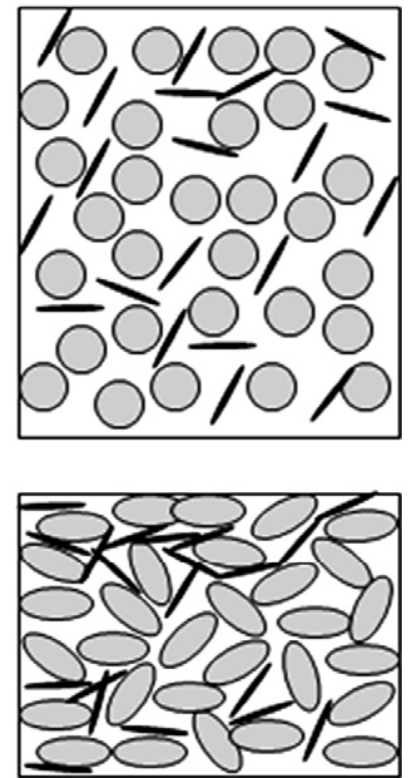

Fig. 10. Scheme of compaction of PA66 particles with CNF at different polymer grain size. (A) $450 \mu \mathrm{m}$ and (B) $100 \mu \mathrm{m}$.

the space available to form conductive paths, and pushing CNF into the interstitial spaces. Under this circumstance, composites present a segregated structure of $\mathrm{CNF}$, which are located on the surface of the polymer, coating the particles, instead of being randomly distributed in the whole system. Although for very different systems, a quite similar behavior has been reported [18,19]. This arrangement is supposed to act as a footprint during the compression molding as it is shown schematically in Fig. 10. Another important aspect worthy to be mentioned is the fact that when the grain size is larger, the percolation threshold is significantly lower. This behavior, which has already been described for other type of systems [18], can be attributed to the fact that when the ratio of the particle size of polymer to nanoadditive is high enough, the coating process is more effective and the arrangements of $\mathrm{CNF}$ in the segregated phase are more appropriate to form the network of connected particles.

\section{Conclusions}

In this work, we carry out the structural characterization as well as the study of electrical properties of systems based on PA66 and CNF and prepared by an alternative procedure to melt processing. The method consists of the physical mixture of the polymer matrix, in the powder form, and the nanoadditive, by a vortex mixer. The study was performed using two polymer particle sizes. From the results, it can be inferred that this method yields materials without significant variation in their structural characteristics, being all composites similar to neat PA66. Regarding electrical conductivity, the materials based on both particle sizes have good electrical conductivity and show lower percolation thresholds than those composites processed from the molten state. One explanation for that fact could be that PA66 solid particles create excluded volume and push CNF into the interstitial space. Under this circumstance, CNF form a segregated distribution of the nanoadditive, which ultimately act as a precursor for the formation of the physical network between particles. However, there are differences between both particle sizes. In spite of the final conductivity value reached by both systems is of the same order, the percolation threshold is lower in the case of the highest polymer particle size. The reason could be that in the case of the highest particle size, the coating process of polymer particles by carbon nanofibers is more effective and the arrangements of CNF in the segregated phase are more appropriate to form the network of connected particles, which is characteristic of percolative behavior.

\section{Acknowledgment}

This work has been carried out with the financial support of MICINN (PET2007_0186_03 and MAT2009-07789).

\section{References}

[1] Huang JC. EMI shielding plastics - a review. Adv Polym Technol 1995; $14: 137-50$.

[2] Stauffer D. Introduction to percolation theory. London: Taylor\&Francis; 1985.

[3] Lin B, Sundararaj U, Pötschke P. Melt mixing of polycarbonate with multiwalled carbon nanotubes in miniature mixers. Macromol Mater Eng 2006;291:227-38.

[4] Procedimiento de obtención de materiales compuestos como conductores eléctricos, ES1641.770.

[5] Vera-Agullo J, Varela-Rizo H, Conesa JA, Almansa C, Merino C, Martin-Gullon I. Evidence for growth mechanism and helix-spiral cone structure of stacked-cup carbon nanofibers. Carbon 2007;45:2751-8.

[6] Ho J, Wei K. Induced $\gamma \rightarrow \alpha$ crystal transformation in blends of polyamide 6 and liquid crystalline copolyester. Macromolecules 2000;33:5181-6.

[7] Jonscher AK. The universal dielectric response. Nature 1977;267:673-9.

[8] Kirkpatrick S. Percolation and conduction. Rev Mod Phys 1973;45:574-88.

[9] Kilbribe BE, Coleman JN, Fraysse J, Fournet P, Cadek M, Drury A, et al. Experimental observation of scaling laws for alternating current and direct current conductivity in polymer-carbon nanotube composite thin films. J Appl Phys 2002;92:4024-30.

[10] Ezquerra TA, Kulescza M, Baltá-Calleja. Electrical transport in polyethylenegraphite composite materials. Synth Metal 1991;41-43:915-20.

[11] Flandin L, Verdier M, Boutherin B, Brechet Y, Cavaillé J-Y. A 3-D numerical simulation of AC electrical properties of short fiber composites. J Polym Sci: Part B: Polym Phys 1999;37:805-14.

[12] Dyre JC, Schroder TB. Universality of ac conduction in disordered solids. Rev Mod Phys 2000;72:873-92.

[13] Bergman DJ, Imry Y. Critical behavior of the complex dielectric constant near the percolation threshold of a heterogeneous material. Phys Rev Lett 1977;39:1222-5.

[14] Laibowitz RB, Gefen Y. Dynamic scaling near the percolation threshold in thin Au films. Phys Rev Lett 1984;53:380-3.

[15] Gefen Y, Aharony A, Alexander S. Anomalous diffusion on percolating clusters. Phys Rev Lett 1983;50:77-80.

[16] Linares A, Canalda JC, Cagiao ME, García-Gutiérrez MC, Nogales A, MartínGullón I, et al. Broad-band electrical conductivity of high density polyethylene nanocomposites with carbon nanoadditives: multiwall carbon nanotubes and carbon nanofibers. Macromolecules 2008;41:7090-7.

[17] McClory C, Bien D, Moore I, Millar B, Davidson J, Carrol T. Recycled carbon fiber filled polyethylene composites. J Appl Polym Sci 2008;107:2015-21.

[18] Malliaris A, Turner DT. Influence of particle size on the electrical resistivity of compacted mixtures of polymeric and metallic powders. J Appl Phys 1971;42:614-8.

[19] Zhao Z, Zheng W, Yu W, Long B. Electrical conductivity of poly(vinylidene fluoride)/carbon nanotubes composites with spherical substructure. Carbon 2009;47:2118-20.

[20] Bortz DR, Merino C, Martín-Gullón I. Carbon nanofibers enhance the facture toughness and fatigue performance of a structural epoxy systems. Compos Sci Technol 2011;71:31-8.

[21] Bauhofer W, Kovacs JZ. A review and analysis of electrical percolation in carbon nanotubes polymer composites. Compos Sci Technol 2009;69:1486-98. 